\title{
Cyclooxygenase-2-mediated upregulation of heme oxygenase 1 mitigates the toxicity of deuterium-tritium fusion radiation
}

\author{
XIAOYAO YANG ${ }^{1,2^{*}}$, HUI LIU $^{1 *}$, XU JIANG ${ }^{1,2}$, CHUFENG JIN $^{1}$, ZHAO XU $^{1}$, \\ TAOSHENG $\mathrm{LI}^{1}$, ZHIGANG WANG ${ }^{1}$ and JUN WANG ${ }^{1}$ \\ ${ }^{1}$ Key Laboratory of High Magnetic Field and Ion Beam Physical Biology, Key Laboratory \\ of Neutronics and Radiation Safety, Chinese Academy of Sciences, Hefei, Anhui 230031; \\ ${ }^{2}$ University of Science and Technology of China, Hefei, Anhui 230026, P.R. China
}

Received March 27, 2018; Accepted July 30, 2018

DOI: $10.3892 /$ ijmm.2018.3799

\begin{abstract}
Utilizing the energy released from the nuclear fusion of deuterium with tritium (D-T) may be an important method of supplying energy in the future. The ionizing radiation emitted from nuclear fusion is a potential health risk to humans, including scientists who are currently performing nuclear fusion experiments and the employees of fusion nuclear plants, in the future. However, there have been few reports on the biological effects of fusion radiation. In the present study, using the High Intensity D-T Fusion Neutron Generator, the DNA damage and its regulation in normal human fibroblasts exposed to fusion radiation were investigated. Heme oxygenase 1 (HO-1), which is reported to induce anti-inflammatory activity, was upregulated in the irradiated cells. Pretreatment with the HO-1 inhibitor, protoporphyrin IX zinc (II), exacerbated double strand break formation following exposure to fusion radiation. The expression of cyclooxygenase-2 (COX-2) contributed to the upregulation of HO-1, as demonstrated by the result that its inhibitor, NS-398, inhibited the induction of HO-1 in irradiated cells. It was further clarified that the ataxia telangiectasia mutated DNA damage response was activated and it stimulated the phosphorylation of $\mathrm{p} 38$ mitogen-activated protein kinase, which was responsible for the upregulation of COX-2 and $\mathrm{HO}-1$. These results provide novel information on fusion radiation-induced biological effects and potential targets for decreasing the associated health risks.
\end{abstract}

Correspondence to: Dr Jun Wang, Key Laboratory of High Magnetic Field and Ion Beam Physical Biology, Laboratory of Neutronics and Radiation Safety, Chinese Academy of Sciences, 350 Shushanhu Road, Hefei, Anhui 230031, P.R. China

E-mail: wangjun0457@ipp.ac.cn

*Contributed equally

Key words: fusion radiation, heme oxygenase 1, cyclooxygenase-2, ATM DNA damage response

\section{Introduction}

Exposure to ionizing irradiation produces DNA damage in cells, including DNA single strand breaks (SSBs) and double strands breaks (DSBs). While the majority of the SSBs can be repaired, DSBs are particularly hazardous to the cell due to the possibility of them resulting in rearrangement of the genome, which is lethal to the cell (1). Radiation induces the upregulation of proteins, including nuclear factor $\kappa$-light-chain-enhancer of activated $\mathrm{B}$ cells $(\mathrm{NF}-\kappa \mathrm{B})$, inducible nitric oxide synthases, tumor necrosis factor- $\alpha$, interleukin-1 (IL-1) and IL-6, which are associated with inflammatory responses; additionally, this may result in DNA damage and damage to non-targeted tissues, and result in increased risk of carcinogenesis (2).

Heme oxygenase 1 (HO-1), an antioxidant enzyme that exhibits low basal expression levels in the majority of cells and tissues, is notably upregulated by a variety of oxidative stress stimuli. The upregulation of HO-1 is generally considered to be an adaptive cellular response against the toxicity of oxidative stress, and has been recognized to exhibit important anti-inflammatory functions (3-5). Previous studies have demonstrated that the targeted overexpression of HO-1 demonstrated beneficial effects in various experimental animal models of inflammation (6,7). The upregulated gene expression of HO-1 is mediated by a network of signaling pathways, among which mitogen-activated protein kinases (MAPKs) serve a primary role (8). Cyclooxygenase-2 (COX-2), the inducible form of COX, is activated by growth factors and cytokines in order to catalyze the conversion of arachidonic acid to prostaglandins (9). The activity of COX-2 is associated with reactive oxygen species production and inflammatory signs in cells, which are important in mediating radiation-induced biological effects (10). The expression of COX-2 can be activated by ionizing radiation and its upregulation is indicated to be associated with the decreased sensitivity of cells to radiation (11-13). As HO-1 and COX-2 contribute to radiation protection and are involved in the equilibrium of oxidative stress, it is hypothesized that interconnections exist between them in irradiated cells, which requires investigation.

The nuclear fusion of deuterium with tritium (D-T) releases notable energy, and investigations into using fusion for the production of electricity has been pursued for decades. 
A number of experimental fusion reactors are under development globally. As fusion reaction produces large quantities of ionizing radiation, including high energy neutron and photon, biological investigations associated with the health risks of fusion radiation are important and necessary for the benefit of scientists and workers (14). In the present study, whether HO-1 and COX-2 are involved in the regulation of damage caused by fusion radiation was investigated, and their upstream regulators were identified.

\section{Materials and methods}

Chemicals. KU55933 was purchased from Santa Cruz Biotechnology, Inc. (Dallas, TX, USA), SB203580 was purchased from Selleck Chemicals (Houston, TX, USA), NS-398 was purchased from Abcam (Cambridge, UK) and protoporphyrin IX zinc (II) (ZnPP) was purchased from Sigma-Aldrich (Merck KGaA, Darmstadt, Germany). These chemicals were dissolved in $100 \%$ dimethyl sulfoxide and stored in small aliquots at $20^{\circ} \mathrm{C}$. Protease inhibitor cocktail and protein inhibitor cocktail were purchased from Roche Diagnostics GmbH (Mannheim, Germany) and Sigma-Aldrich (Merck KGaA), respectively.

Cell culture and radiation. Normal human lung fibroblasts (NHLFs), an adherently grown human primary lung fibroblast, were purchased from BeNa Culture Collection (Beijing, China). The NHLF cells were cultured in Dulbecco's modified Eagle's medium/F12 (GE Healthcare Life Sciences, Little Chalfont, UK) with $10 \%$ fetal bovine serum (Clark Bioscience, Richmond, VA, USA) and grown in a humidified $5 \% \mathrm{CO}_{2}$ incubator at $37^{\circ} \mathrm{C}$. The neutron radiation appliance was a High Intensity D-T Fusion Neutron Generator, which was developed at the Institute of Nuclear Energy Safety Technology, Chinese Academy of Sciences (Hefei, China) by the Fusion Design and Study team. The production and the property of the fusion neutron were described previously by Wu (15). Radiation doses $(0,0.01,0.12,0.6$, and 1.2 Gy) were adjusted by setting the cells at different linear distances from the neutron source.

Immunofluorescence staining of $\gamma-H_{2}$ A histone family member $X\left(\gamma-H_{2} A X\right)$. The NHLF cells were cultured on $0.17 \mathrm{~mm}$-thick glass-bottom cell culture dishes and exposed to neutron radiation. At the desired time points $(0,0.5,1,3,6$, and $12 \mathrm{~h})$, the cells were washed with PBS and fixed with $4 \%$ paraformaldehyde at room temperature for $30 \mathrm{~min}$. Subsequently, the cells were washed with PBS and permeated with $0.5 \%$ Triton X-100 at room temperature for $30 \mathrm{~min}$. Following blocking with PBST ( $0.1 \%$ Triton $\mathrm{X}-100)$ containing $1 \%$ bovine serum albumin (BSA; Sangon Biotech Co., Ltd., Shanghai, China) and $0.1 \%$ Triton X-100 at $37{ }^{\circ} \mathrm{C}$ for $1 \mathrm{~h}$, the cells were incubated with anti- $\gamma-\mathrm{H}_{2} \mathrm{AX}$ antibody (1:400; cat. no. 2577; Cell Signaling Technology, Inc., Danvers, MA, USA), which was diluted in PBST containing $1 \% \mathrm{BSA}$ at $4^{\circ} \mathrm{C}$ overnight with gentle agitation. The dishes were then washed three times with PBST for 5 min. Alexa Fluor-594-conjugated goat anti-rabbit IgG (1:800; cat. no. 111-585-003; Jackson ImmunoResearch Laboratories, Inc., West Grove, PA, USA) diluted in PBST containing 1\% BSA was added into the samples. Following incubation for
$2 \mathrm{~h}$ at room temperature, the cells were washed three times with PBST for 15 min and counterstained with Hoechst 33342. ( $2 \mu \mathrm{g} / \mathrm{ml})$ Images were captured under an Olympus IX83 fluorescence microscope using a 40X air-objective (Olympus Corporation, Tokyo, Japan) and analyzed with Image J1.49 software (National Institutes of Health, Bethesda, MD, USA). For $\gamma$-H2AX quantification, images captured in the Hoechst 33342 channel were used to define the nuclear region. The fluorescence intensity of $\gamma-\mathrm{H} 2 \mathrm{AX}$ in the defined nuclear region was measured to reflect the levels of DNA DSB. At least 500 cells were analyzed for each sample.

Immunoblot analysis. The cells were then washed twice with PBS and total cell lysate was prepared with radioimmunoprecipitation assay buffer containing protease inhibitors and protein phosphatase inhibitors. Protein concentrations were determined using a BCA kit (Sangon Biotech Co., Ltd.). The cell lysate $(50 \mu \mathrm{g})$ was resolved using $10 \%$ or $12 \%$ SDS-PAGE and transferred onto a polyvinylidene fluoride (PVDF) membrane. Following blocking in tris-buffered saline with $0.1 \%$ Tween 20 with $1 \%$ skim milk, the PVDF membrane was incubated with primary antibodies at $4^{\circ} \mathrm{C}$ overnight. The primary antibodies used were as follows: $\alpha$-tubulin (1:20,000; cat. no. ab108629; Abcam); phosphorylated (p)-ataxia telangiectasia mutated (p-ATM; 1:1,000; cat. no. DR1002; Merck KGaA); Cox-2 (1:1,000; cat. no. 160112; Cayman Chemical Company, Ann Arbor, MI, USA); $\beta$-actin (1:500; cat. no. sc-8432; Santa Cruz Biotechnology, Inc.); p-p38 (1:1,000; cat. no. 612280; BD Biosciences, Franklin Lakes, NJ, USA); and HO-1 (1:1,000; cat. no. 10701; ProteinTech Group, Inc., Chicago, IL, USA). Subsequently, the membrane was washed three times with PBS with $0.1 \%$ Tween 20 and incubated with corresponding horseradish peroxidase-conjugated secondary antibodies (goat anti-rabbit, cat. no. 111-035-003 or goat anti-mouse, cat. no. 115-035-003; both 1:200,000; Jackson ImmunoResearch Laboratories, Inc.) for $2 \mathrm{~h}$ at room temperature. The protein bands were visualized using enhanced chemiluminescent substrate (Wuhan Boster Biological Technology, Ltd., Wuhan, China).

Reverse transcription-quantitative polymerase chain reaction (RT-qPCR) analysis. Total RNA (100 ng/ $\mu \mathrm{l})$ was extracted using RNAiso reagent (Takara Bio, Inc, Otsu, Japan). The total RNA was added into the reaction mixture from One-Step SYBR $^{\circledR}$ PrimeScript ${ }^{\mathrm{TM}}$ PLUS RT-PCR kit. The reaction mixture was as follows: Reaction volume, $20 \mu \mathrm{l} ; 2 \mathrm{X}$ One Step SYBR RT-PCR Buffer, $10 \mu \mathrm{l}$; Takara Ex Taq HS Mix, $1.2 \mu \mathrm{l}$; PrimeScript PLUS RTase Mix, $0.4 \mu \mathrm{l}$; Forward Primer $(10 \mu \mathrm{M}), 0.8 \mu \mathrm{l}$; Reverse Primer $(10 \mu \mathrm{M}), 0.8 \mu \mathrm{l}$; Rox Reference Dye, $0.4 \mu \mathrm{l}$; Total RNA, $2 \mu \mathrm{l}$; RNase Free $\mathrm{ddH}_{2} \mathrm{O}, 4.4 \mu \mathrm{l}$. RT-qPCR analysis was performed on a StepOne ${ }^{\text {TM }}$. Real-Time PCR system (Applied Biosystems; Thermo Fisher Scientific, Inc., Waltham, MA, USA) according to $2^{-\Delta \Delta \mathrm{Cq}}$ method (16). Reverse transcription was performed at $42^{\circ} \mathrm{C}$ for $10 \mathrm{~min}$. The PCR conditions were as follows: $95^{\circ} \mathrm{C}$ for $1 \mathrm{~min}, 50^{\circ} \mathrm{C}$ for $30 \mathrm{sec}, 72^{\circ} \mathrm{C}$ for $30 \mathrm{sec}, 35$ cycles. The primers for HO-1 were: Forward 5'-CTGTGTAACCTCTGCTGTTCC-3' and reverse 5'-CCACACTACCTGAGTCTACC-3'. The primers for human $\beta$-actin were: Forward 5'-CCTGGCACCCAGCACAAT-3' and reverse 5'-GGGCCGGACTCGTCATAC-3'. 

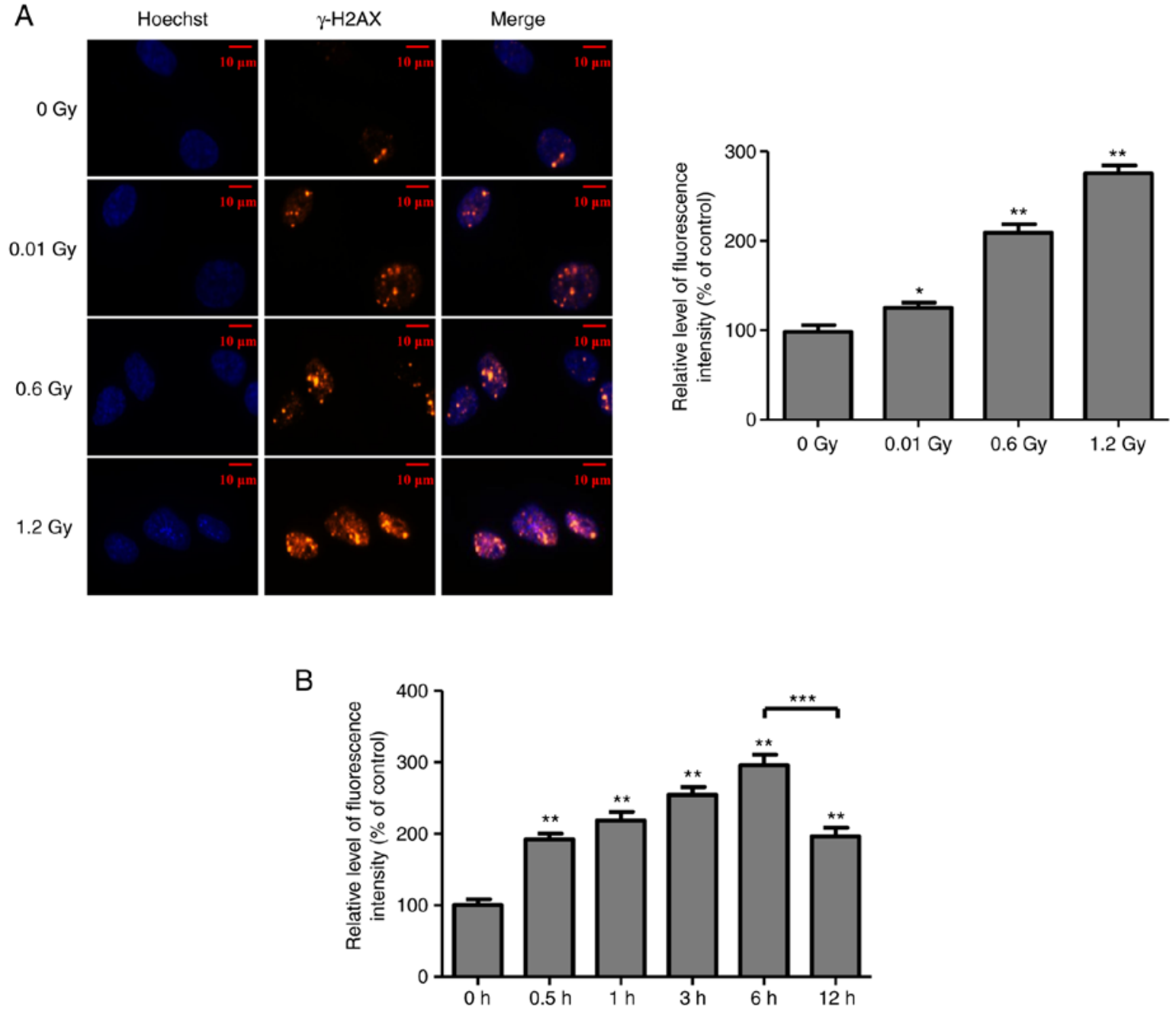

Figure 1. Induction of DNA double strands breaks in normal human lung fibroblast cells exposed to fusion radiation. (A) Cells were exposed to 0.01, 0.6 and 1.2 Gy $14.1 \mathrm{MeV}$ neutrons. After $1 \mathrm{~h}$, the cells were fixed and immunofluorescent staining of $\mathrm{H}_{2} \mathrm{AX}$ (pSer139) was performed (scale bar=10 $\mu$ m). (B) Residual expression levels of $\mathrm{H}_{2} \mathrm{AX}$ (pSer139) in cells exposed to $0.6 \mathrm{~Gy} 14.1 \mathrm{MeV}$ neutrons at different time points of exposure. ${ }^{*} \mathrm{P}<0.05$, ${ }^{* *} \mathrm{P}<0.01$, ${ }^{* * * *} \mathrm{P}<0.001$.

Statistical analysis. All data are presented as the mean \pm standard deviation of at least three independent experiments performed in triplicate. Statistical significance between two groups was evaluated using Student's t-test with GraphPad Prism 5 (GraphPad Software, Inc., San Diego, CA, USA). Statistical significance between multiple groups was evaluated using one-way analysis of variance with SPSS 12.0 software (SPSS, Inc., Chicago, IL, USA). P $<0.05$ was considered to indicate a statistically significant difference.

\section{Results}

Induction of DNA DSBs by neutron radiation. The induced levels of DNA DSBs in the irradiated NHLF cells were evaluated to assess the toxicity of D-T neutron radiation. The cells were exposed to $0.01,0.6$ and 1.2 Gy neutrons and, $1 \mathrm{~h}$ later, an immunofluorescence staining assay was performed to analyze the DSB levels. As shown in Fig. 1A, there was a dose-dependent tendency for the induction of DSB in the dose range used in the experiment. Subsequently, the levels of DSBs at different time intervals following 0.6 Gy neutron radiation were detected (Fig. 1B). A significant increase in the number of DSBs was observed 30 min following radiation exposure, and enhanced DSB formation was sustained to the $6 \mathrm{~h}$ time point. At $12 \mathrm{~h}$ post-radiation, the DSB levels decreased, reflecting the repair of neutron-induced DNA damage.

Upregulation of HO-1-alleviates neutron-induced DSBs. HO-1 serves important biological roles in maintaining cellular oxidative equilibrium and has been reported to protect cells against stress induced damage; therefore, whether neutron radiation stimulated the expression of HO-1was determined. Following 6 or $12 \mathrm{~h}$ of exposure to 0.6 Gy neutrons, the NHLF cells were collected and the expression of HO-1 in total cell lysates was detected by western blot analysis. As shown in Fig. 2A, upregulation of HO-1 was observed $6 \mathrm{~h}$ post-radiation. At the 12-h time point, the expression level of HO-1 was enhanced by $75 \%$, compared with that in the control. Subsequently, the effect of HO-1 on radiation-induced DSB formation was determined. Pretreatment with Znpp increased DSB formation by $\sim 30 \%$ in the 0.6 Gy neutron-irradiated cells (Fig. 2B). These results demonstrated that induction of the expression of $\mathrm{HO}-1$ by neutron radiation alleviated DNA damage.

COX-2 mediates the upregulation of $\mathrm{HO}-1$ in irradiated cells. COX-2 has been reported to be upregulated in ionizing irradiated 
A
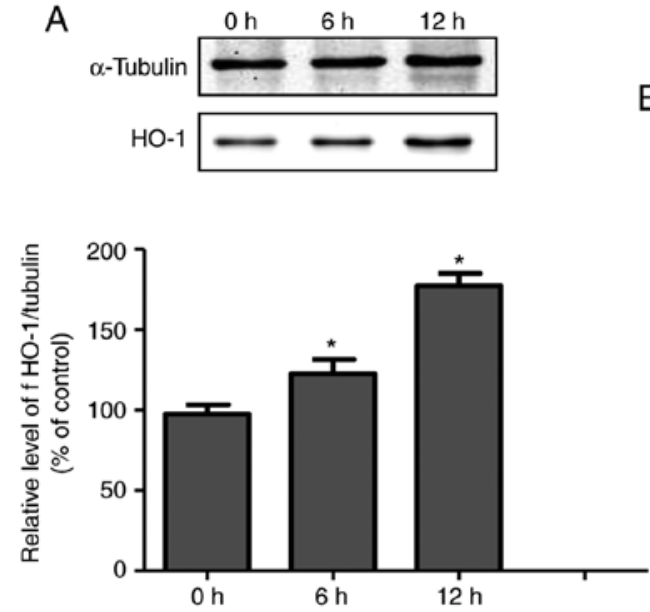

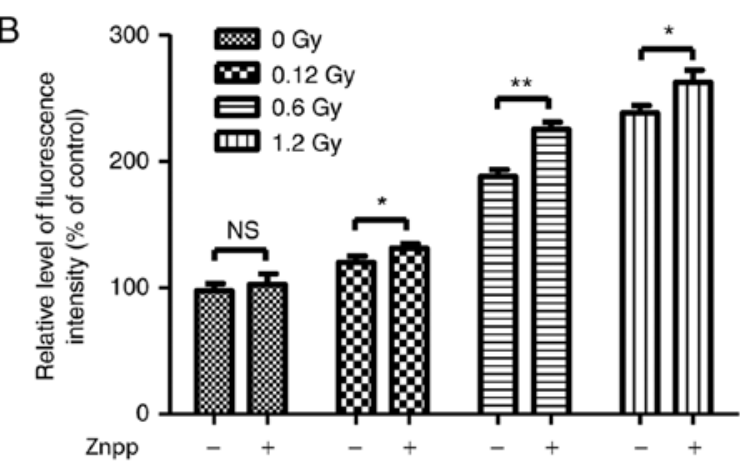

Figure 2. Upregulation of the expression of HO-1 by fusion radiation. (A) Cells were exposed to 0.6 Gy fusion neutron. At the indicated time points, the irradiated cells were collected and the expression levels of HO-1 were determined by western blot analysis. (B) Cells were pre-incubated with HO-1 inhibitor $\mathrm{ZnPP}$ (final concentration, $2.5 \mu \mathrm{M}$ ) for $1 \mathrm{~h}$ prior to fusion radiation exposure. At $1 \mathrm{~h}$ following exposure to radiation, the cells were fixed for $\mathrm{H}_{2} \mathrm{AX}$ ( $\mathrm{pSer139}$ ) immunostaining. ${ }^{*} \mathrm{P}<0.05,{ }^{* *} \mathrm{P}<0.01$. HO-1, heme oxygenase 1; ZnPP, protoporphyrin IX zinc (II); NS, not significant.
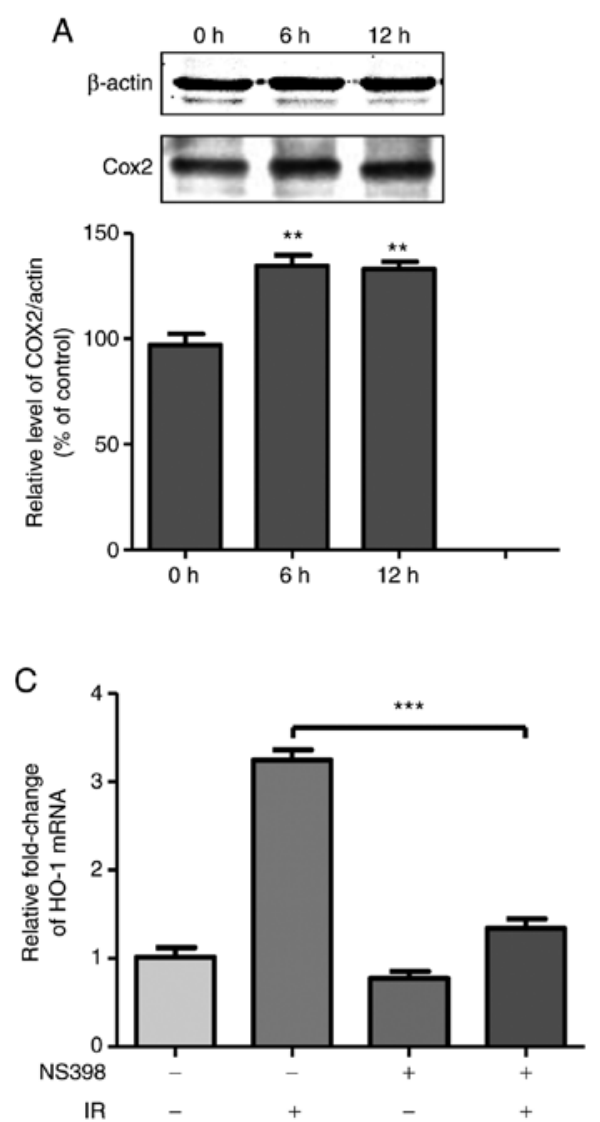

B
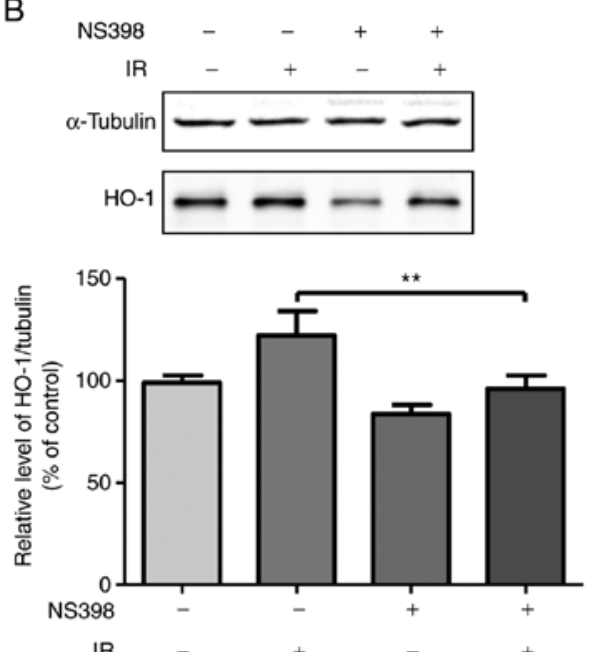

D

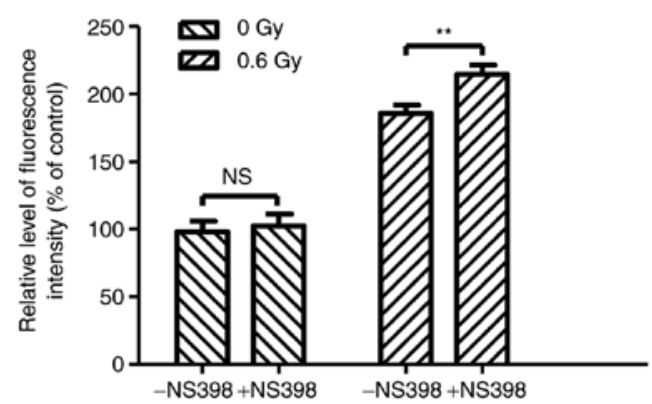

Figure 3. Upregulation of COX-2 by fusion radiation promotes the expression of HO-1. (A) Cells were exposed to 0.6 Gy fusion neutron. At the indicated time points, the irradiated cells were collected and the expression levels of COX-2 were determined by western blot analysis. Cells were treated with COX-2 inhibitor NS-398 (final concentration, $40 \mu \mathrm{M}$ ) for $1 \mathrm{~h}$ prior to fusion radiation exposure. At $6 \mathrm{~h}$ following exposure to radiation, (B) protein and (C) mRNA levels of HO-1 were detected by western blot and reverse transcription-quantitative polymerase chain reaction analyses, respectively. (D) Cells were treated with COX-2 inhibitor NS-398 (final concentration, $40 \mu \mathrm{M}$ ) for $1 \mathrm{~h}$ prior to fusion radiation exposure. At $1 \mathrm{~h}$ following exposure to radiation, the cells were fixed for $\mathrm{H}_{2} \mathrm{AX}$ (pSer139) immunostaining. ${ }^{* *} \mathrm{P}<0.01,{ }^{* * *} \mathrm{P}<0.001$. COX-2, cyclooxygenase 2; HO-1, heme oxygenase 1; IR, irradiation; NS, not significant.

cells and the inhibition of COX-2 results in enhanced toxicity; therefore, whether COX-2 affected the expression of HO-1 in neutron irradiated cells was investigated. The expression level of COX-2 was detected at 6 and $12 \mathrm{~h}$ following radiation expo- sure. As shown in Fig. 3A, COX-2 was upregulated by $30 \%$ at $6 \mathrm{~h}$ compared to $0 \mathrm{~h}$ in the irradiated cells. Subsequently, the protein and mRNA levels of HO-1 were detected in irradiated cells treated with COX-2 inhibitor NS-398. The cells were 
A
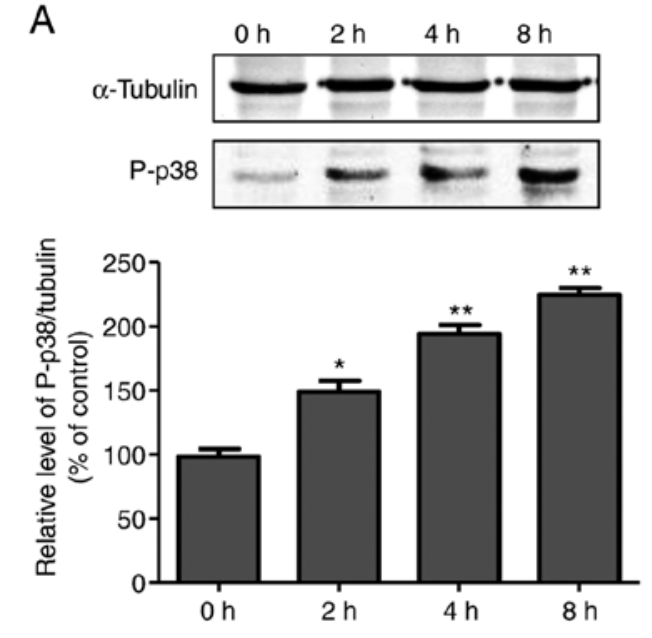

C
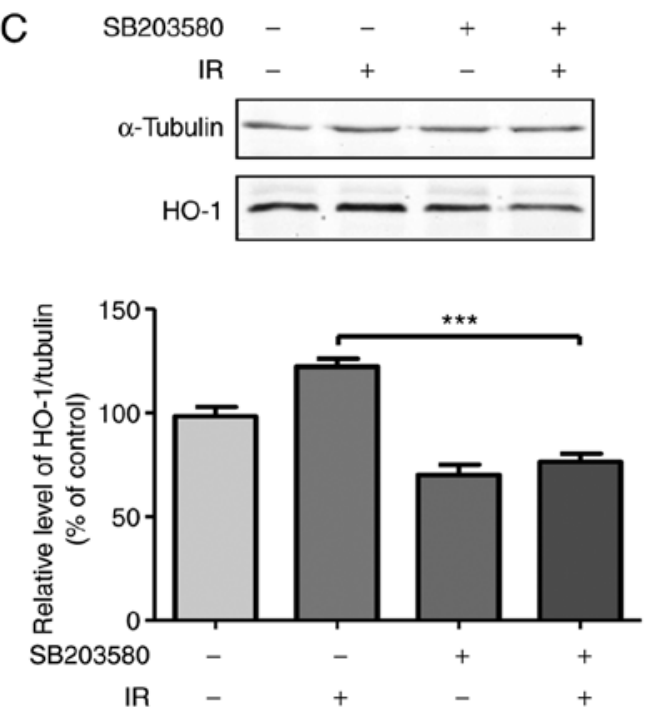

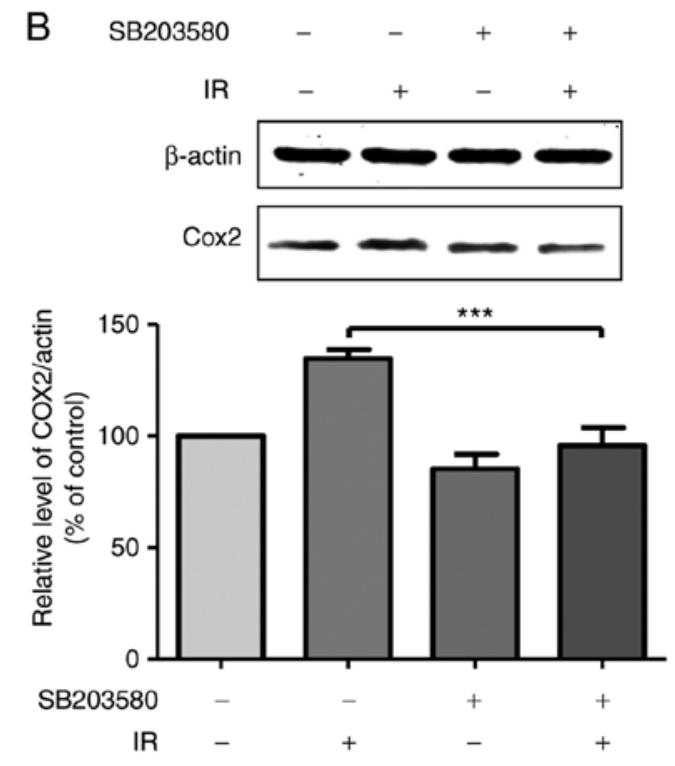

D

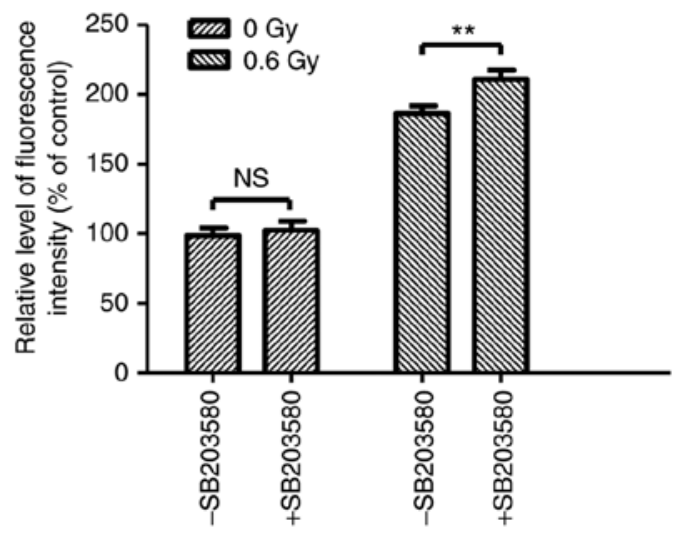

Figure 4. Activation of p38 MAPK contributes to the upregulation of COX-2 and HO-1 in cells exposed to fusion radiation. (A) Cells were exposed to 0.6 Gy fusion neutron. At the indicated time points, irradiated cells were collected and the expression levels of phosphorylated p38 were determined by western blot analysis. Cells were pre-incubated with p38 inhibitor SB203580 (final concentration, 20 $\mu \mathrm{M}$ ) for $1 \mathrm{~h}$ prior to fusion radiation exposure. At $6 \mathrm{~h}$ following exposure to radiation, cells were collected. The expression levels of (B) COX-2 and (C) HO-1 were detected by western blot analysis. (D) Cells were pre-incubated with p38 inhibitor SB203580 (final concentration, $20 \mu \mathrm{M}$ ) for $1 \mathrm{~h}$ prior to fusion radiation exposure. At $1 \mathrm{~h}$ following exposure to radiation, the cells were fixed for $\mathrm{H}_{2} \mathrm{AX}$ (pSer139) immunostaining. ${ }^{*} \mathrm{P}<0.05,{ }^{* *} \mathrm{P}<0.01,{ }^{* * *} \mathrm{P}<0.001$. COX-2, cyclooxygenase 2; HO-1, heme oxygenase 1; p-p38, phosphorylated p38; IR, irradiation; NS, not significant.

pre-incubated with NS-398 for $1 \mathrm{~h}$ prior to irradiation. As shown in Fig. 3B and C, pretreatment with NS-398 inhibited the induced expression of HO-1 in the neutron-irradiated NHLF cells. These results indicated that COX-2 was an important mediator for the upregulation of HO-1 in irradiated cells. The effect of COX-2 on radiation-induced DSB formation was then investigated. Treatment of the NHLF cells with COX-2 inhibitor NS-398 increased neutron-induced DSB formation (Fig. 3D). The DSB formation rate increased by $\sim 15 \%$ in the cells pretreated with NS-398 and then exposed to 0.6 Gy neutron irradiation. These results demonstrated that the induction of the expression of COX-2 by neutron radiation alleviated DNA damage.

COX-2/HO-1 is regulated by p38 in irradiated cells. p38, a member of the MAPKs, is reported to be involved in ionization radiation-induced biological responses. To determine the upstream regulator of $\mathrm{COX}-2$, phosphorylation was first determined. As shown in Fig. 4A, the phosphorylation of p38 showed a tendency to increase following radiation exposure, to a level of 1.5-fold at the $2 \mathrm{~h}$ time point and 2.2-fold at the $8 \mathrm{~h}$ time point, compared with the control group. To determine the association between the activation of $\mathrm{p} 38$ and the expression of COX-2, the cells were treated with p38 inhibitor SB203580 for $1 \mathrm{~h}$ prior to radiation exposure. At $6 \mathrm{~h}$ post-radiation exposure, the expression levels of COX-2 and HO-1 in the total cell lysate were detected and are shown in Fig. $4 \mathrm{~B}$ and $\mathrm{C}$. The results demonstrated that the induction of COX-2 and HO-1 was significantly suppressed by SB203580, indicating that the activation of $\mathrm{p} 38$ by radiation exposure contributed to the induced expression of COX-2 and HO-1. Subsequently, the expressions levels of DSB formation in the irradiated cells treated with p38 inhibitor SB203580 were detected. Preincubation with 
A
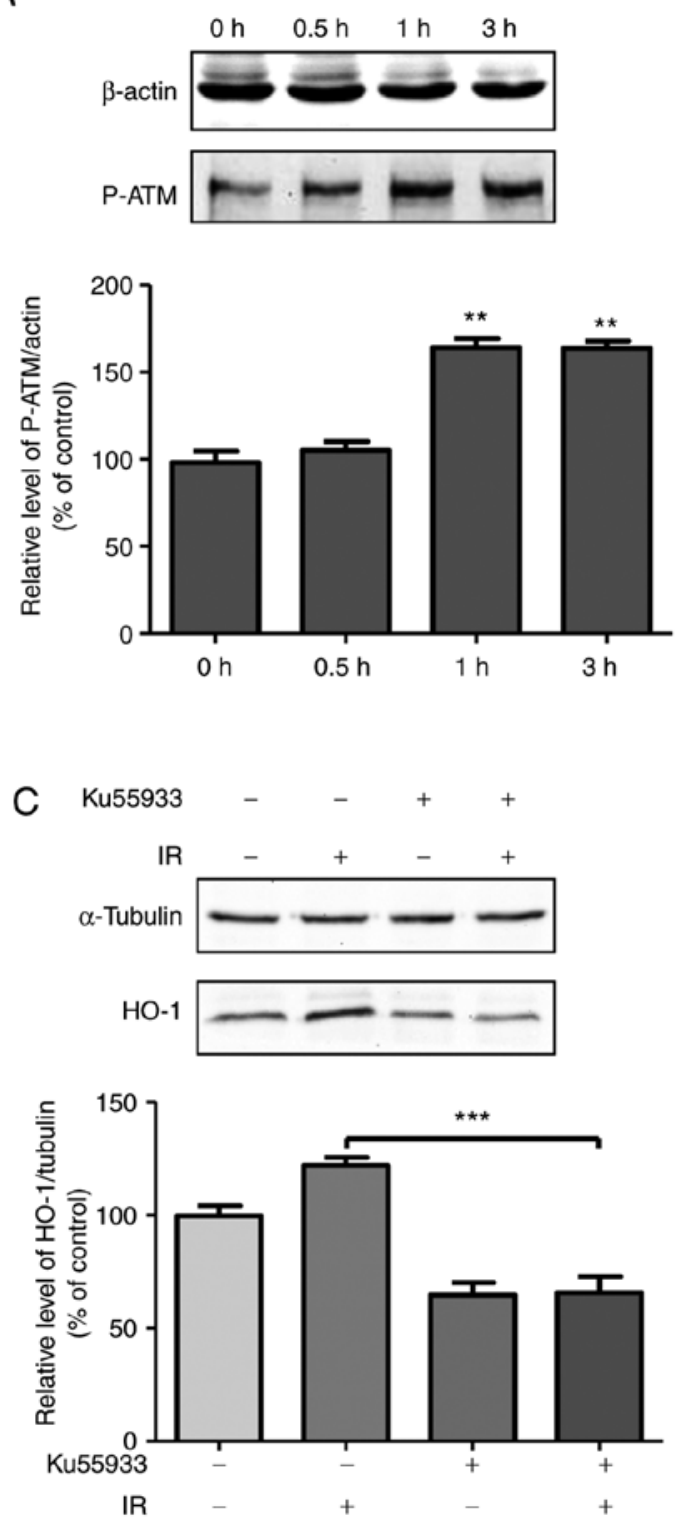
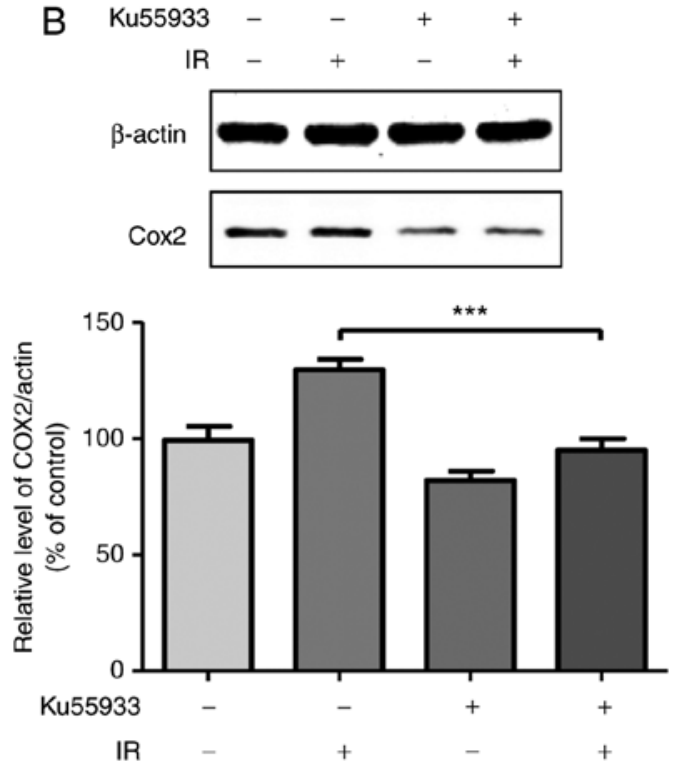

D Ku55933 $\quad-\quad-\quad+\quad+$
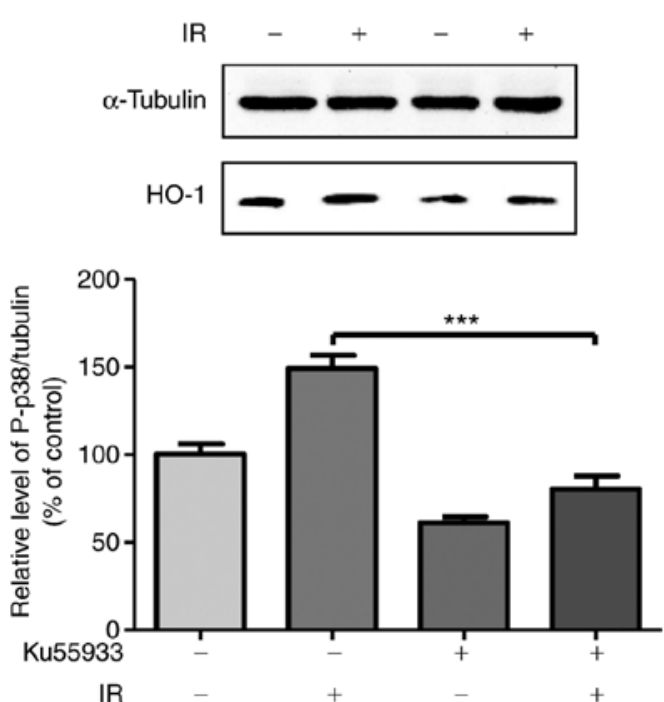

Figure 5. ATM DNA damage response is responsible for the upregulation of p-p38/COX-2/HO-1. (A) Cells were exposed to 0.6 Gy fusion neutron. At the indicated time points, irradiated cells were collected, and the expression levels of phosphorylated ATM were determined by western blot analysis. Cells were treated with ATM inhibitor KU55933 (final concentration, $25 \mu \mathrm{M}$ ) for $0.5 \mathrm{~h}$ prior to fusion radiation exposure. At $6 \mathrm{~h}$ following exposure to radiation, the cells were collected and the expression levels of (B) COX-2 and (C) HO-1 were detected by western blot analysis. (D) At 2 h following pretreatment with $25 \mu \mathrm{M}$ KU55933 and fusion radiation, cells were collected and the levels of p-p38 were determined by western blot analysis. ${ }^{* *} \mathrm{P}<0.01,{ }^{* * *} \mathrm{P}<0.001$. ATM, ataxia telangiectasia mutated; COX-2, cyclooxygenase 2; HO-1, heme oxygenase 1 ; IR, irradiation.

SB203580 increased the DSB formation rate by $\sim 13 \%$ in the 0.6 Gy neutron-irradiated cells (Fig. 4D). These results demonstrated that the induction of the expression of p38 by neutron radiation alleviated DNA damage.

ATM DNA damage response stimulates $p$-p38/COX-2/HO-1. ATM is a DNA damage response kinase and it is rapidly activated through autophosphorylation in irradiated cells. Following neutron radiation exposure, the phosphorylation of ATM was observed at $30 \mathrm{~min}$ and was enhanced up to $3 \mathrm{~h}$ post-radiation exposure (Fig. 5A). Subsequently, the ATM inhibitor, KU55933, was used to investigate whether the enhanced expression of COX-2 and HO-1 was due to the activation of ATM. The cells were treated with KU55933 for $0.5 \mathrm{~h}$ prior to irradiation, and at $6 \mathrm{~h}$ post-radiation, the levels of COX-2 and HO-1 were detected. As shown in Fig. 5B and $\mathrm{C}$, the radiation-induced expression of COX-2 and HO-1 was suppressed, indicating the ATM-regulated DNA damage response contributed to COX-2- and HO-1-associated protection against neutron-induced toxicity. Furthermore, the phosphorylation of p38 following treatment with KU55933 in irradiated cells was identified, which is shown in Fig. 5D. In irradiated cells pretreated with KU55933, the phosphorylation of p38 was significantly inhibited. These results indicated that the activation of $\mathrm{p} 38$ was controlled by ATM in neutron-irradiated cells, and also confirmed the role of $\mathrm{p} 38$ in upregulating the expression of COX-2 and HO-1. 


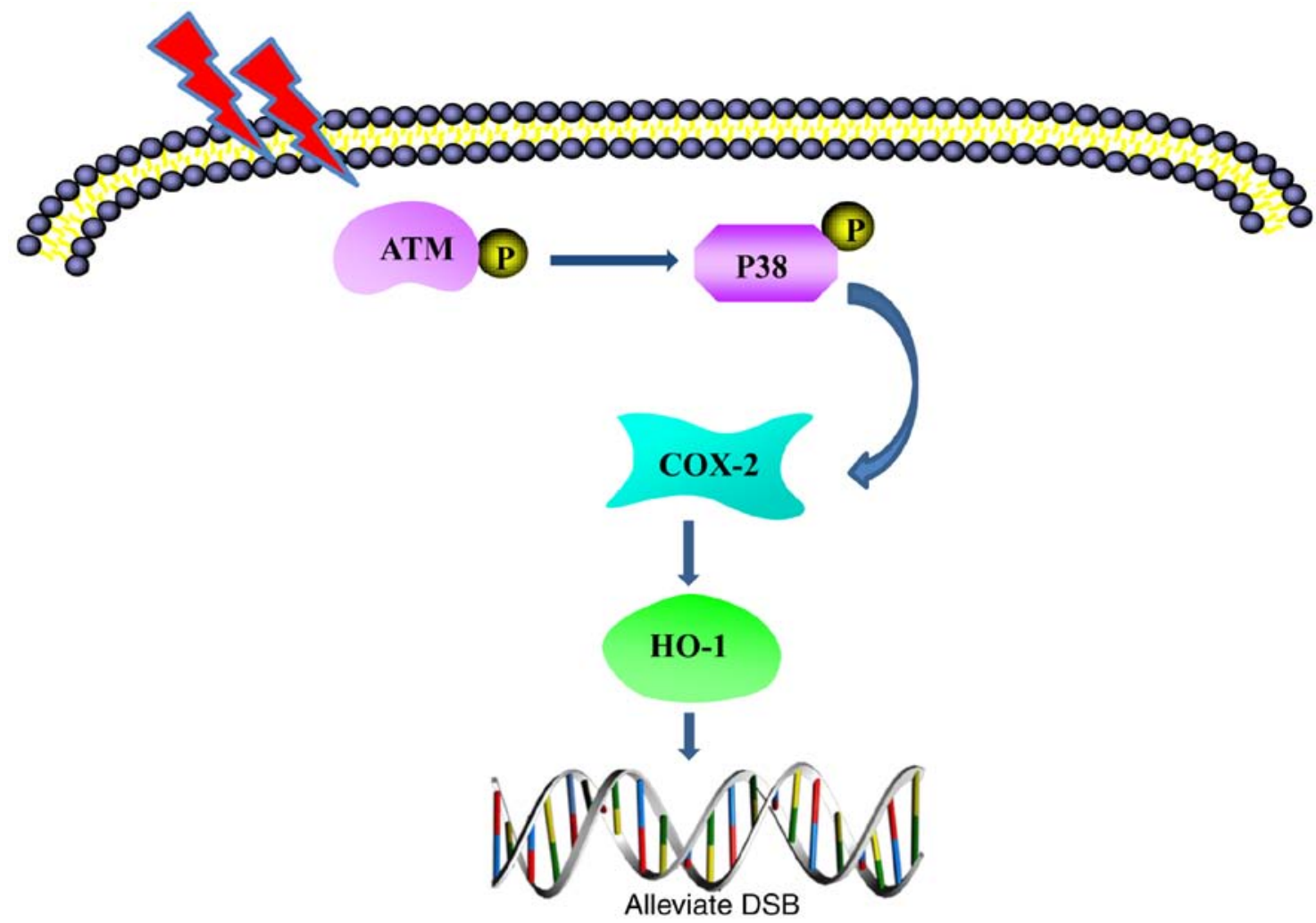

Figure 6. Schematic of the suggested model based on the main outcomes of the present study. ATM, ataxia telangiectasia mutated; COX-2, cyclooxygenase 2; HO-1, heme oxygenase 1; DSB, double strand break.

\section{Discussion}

The notable energy release from the process of D-T nuclear fusion has attracted the attention of nuclear physicists due to its possible significant influence on the methods of supplying energy in the future. The $14.1 \mathrm{MeV}$ neutron produced during the reaction of D-T fusion is a potential health risk due to ionization and damaging tissues; therefore, an understanding of the biological effects induced by the $14.1 \mathrm{MeV}$ neutron is important for protection and medicinal treatment against neutron radiation risks. However, current knowledge on the biological effects and the underlying mechanisms remains limited. In the present study, the level of DNA DSBs, which is a typical DNA damage marker for ionizing radiation, was assessed to detect the level of damage induced by fusion radiation in NHLFs. The results demonstrated that the DSB levels were radiation dose-dependent in the range of 0-1.2 Gy. Compared with gamma-rays, the linear energy transfer of neutrons is notably higher, which means neutron deposits increase energy when traversing cells and causes increased ionization and DSBs (17). Broustas et al (18) compared gene expression profiles in a human peripheral blood model to neutron and X-ray radiation. They identified 125 genes that responded significantly to the two types of radiation as a function of dose, with the magnitude of response to neutrons generally being increased, compared with that observed following X-ray exposure (18). The relative biological effects induced by neutrons varied with the neutron energy. Tanaka et al (19) compared the efficacies of neutron with energy from $0.18-2.30 \mathrm{MeV}$ in the induction of comet DNA and chromosome aberrations. They concluded that $0.37 \mathrm{MeV}$ neutron caused of the most DNA damage, whereas the $2.30 \mathrm{MeV}$ neutron caused the least damage (19); however, the biological effects induced by neutrons with energies $>10 \mathrm{MeV}$, for example, the $14.1 \mathrm{MeV}$ fusion neutron, remain to be fully elucidated. The results of the present study demonstrated that the neutron emitted from D-T fusion was effective in causing DNA damage; therefore, its biological effects warrant further examination.

HO-1, an essential enzyme in heme catabolism, cleaves heme to form biliverdin, carbon monoxide (CO) and ferrous iron. The transcription of HO-1 can be mediated by redox-dependent transcription factors, including NF-E2-related factor 2 (NRF2), NF- $\kappa B$, and activating protein-1 (8). It has been shown to protect cells from various stresses-induced toxicity. HO-1-derived $\mathrm{CO}$ is an anti-inflammatory molecule. A previous study indicated that $\mathrm{CO}$ exposure facilitated the homologous recombination repair of DNA damage through an ATM/ataxia telangiectasia and Rad3-related protein (ATR)-dependent pathway (20). Chen et al (21) reported that, in low dose $\alpha$-particle irradiated A549 cells, HO-1 was upregulated in a NRF2-dependent manner, and its increased expression conferred A549 resistance following high dose $\alpha$-particle radiation. Furthermore, scavenging $\mathrm{CO}$ reduced the resistance to radiation (21). Additionally, in a study on radiation-induced bystander effects, Han et al (22) determined that $\mathrm{CO}$ (released from $\mathrm{CO}$ release molecule 2) treatment resulted in the decreased formation of DSBs in bystander cells. A report by Singh et al (23) demonstrated that treatment with the mixture of podophyllotoxin and rutin reduced the levels of oxidative stress and apoptotic cell death in gamma-ray 
irradiated mouse bone marrow and spleen. Additionally, the NRF2-mediated upregulation of HO-1 partially contributed to the protective role of podophyllotoxin and rutin (23). The results of the present study indicated that the inhibition of HO-1 activity exacerbated fusion radiation-induced DNA damage, confirmed the role of HO-1 in radiation protection, and indicated that the targeted upregulation of HO-1 may be a potential method to decrease the risk of fusion radiation to health.

The expression of COX-2 is upregulated in numerous types of cancer. Furthermore, its product prostaglandin $\mathrm{H} 2$ is converted by prostaglandin E2 (PGE2) synthase into PGE2, which in turn can stimulate cancer progression. Consequently, COX-2 is a target for the development of drugs that prevent and treat cancer. Previous studies have reported that the targeted inhibition of COX-2 improved the efficacy of cancer radiotherapy, indicating the protective role of COX-2 in irradiated tumor cells (9-13). Hofer et al (24) determined in animal experiments that, following gamma-ray radiation, male COX-2-knockout mice exhibited attenuated hematopoiesis, compared with wild-type mice. Additionally, it was concluded that the genetic disruption of COX-2 has a positive effect in hematopoiesis under basal conditions, but is detrimental following radiation exposure (24). Ozbilgin et al (25) indicated that the increased expression of COX-1 and COX- 2 in the urothelium of mice may prevent bladder damage from acute gamma-ray radiation, and benefits the differentiation and restoration of the urothelium. Zuo et al (26) reported that, treatment of mouse epidermal cells with a low concentration of arsenite increased the expression of COX-2, and this induction of COX-2 resulted in decreased levels of apoptosis following ultraviolet B treatment. These data indicated that, although COX-2 is considered to mediate inflammation and promote carcinogenesis, its upregulation is beneficial under a number of stress conditions. To the best of our knowledge, there has been no previous report on the functions of COX-2 in neutron-irradiated cells. The present study indicated that COX-2 may exert a protective effect through stimulating the expression of HO-1, in order to decrease the DSB levels in cells exposed to fusion radiation, which is consistent with the aforementioned data.

ATM is a well-known DNA damage-response protein. In cultured cells, elevated DSBs activate ATM through the autophosphorylation of serine 1981. Additionally, it regulates the functions of proteins involved in cell cycle checkpoints, apoptotic cell death and DNA damage repair, including p53, BRCA1 and nibrin (27). In the present study, the phosphorylation of ATM was enhanced by fusion radiation, which is consistent with previous results observed in cells exposed to other types of ionizing radiation (28). As COX-2 was upregulated, whether its expression was under the control of the activation of ATM following fusion radiation was investigated. Park et al (29) reported that gefitinib, a small-molecular epidermal growth factor receptor tyrosine kinase inhibitor, increased the radiation sensitivity of non-small cell lung cancer cell lines NCI-H460 and VMRC-LCD through inhibiting the activation of ATM. Additionally, the overexpression of COX-2 in NCI-H460 cells reduced the radiation sensitivity induced by gefitinib; however, no direct association was determined between the activation of ATM and expression of COX-2 (29). Chacko et al (30) determined that the hydro-alcoholic extract of Clerodendron infortunatum (CIE) reduces the total body gamma-ray radiation exposure in mice. Additionally, in the intestinal tissue of irradiated animals, following CIE treatment, the expression levels of ATM, but not its phosphorylated form, were elevated; however, the expression of COX-2 was reduced (30). In a previous study by Kim and Pyo (31), it was reported that treatment with the mixture of 17-AAG, an inhibitor of heat shock protein 90, and celecoxib, an inhibitor of COX-2, increased the sensitivity of various human cancer cells to radiation through downregulating ATM and ATR (31). These previous studies indicated that the associations between the activation of ATM and expression of COX-2 are variable in irradiated cells. In the present study, the ATM inhibitor suppressed the upregulation of COX-2 and HO-1, indicating a regulatory role of the ATM DNA response on the expression on COX-2 in cells exposed to fusion radiation. Additionally, it was clarified that $\mathrm{p} 38$ was a mediator of ATM and COX-2. p38 is a member of the MAPKs and responds to a variety of stress stimuli, including cytokines, radiation and heat shock. Its activation in irradiated cells is associated with radiation-induced cell cycle arrest and apoptosis $(32,33)$. Acheva et al (34) used a $3 \mathrm{D}$ organotypic skin model to investigate the mechanisms of radiation-induced inflammatory responses following localized irradiation, and determined that the rapid activation of $\mathrm{NF}-\kappa \mathrm{B}$, phosphorylated $\mathrm{p} 38$ and $\mathrm{COX}-2$ were upregulated in the irradiated and bystander areas of the $3 \mathrm{D}$ cultures (34). However, in a study by Hung et al (35), it was demonstrated that the induction of COX-2 under endoplasmic reticulum stress was controlled by $\mathrm{NF}-\kappa \mathrm{B}$, which was regulated by phosphorylated p38 (35). Furthermore, Tessner et al (36) determined that p38 is critical for the enhanced transcription and expression of COX-2 in gamma-ray-irradiated human epithelial cells (36), which was consistent with the observation by Hung et al (35). Additionally, $\mathrm{Hu}$ et al (37) observed in an in vitro epithelial wounding model that the production of PGE2 was increased in a time-dependent manner via the activation of COX-2, which was stimulated by the phosphorylation of extracellular signal-regulated protein kinase $1 / 2$, another member of the MAPK family (37). The results of the present study demonstrated that COX-2 and its mediated upregulation of HO-1 were regulated by activated p38, and that p38 was also associated with the ATM DNA damage response, which upregulated the expression of COX-2/HO-1 in cells exposed to fusion radiation. These reports indicate that multiple regulatory mechanisms of MAPK members on the expression of COX-2 may be correlated with specific stress conditions. A hypothetic model is depicted in Fig. 6.

In conclusion, the present study demonstrated the involvement of HO-1 in alleviating fusion radiation-induced DNA damage in NHLFs. Induction of the pro-inflammatory protein COX-2 by fusion radiation contributed to the upregulation of HO-1. Furthermore, the ATM DNA damage response was investigated, which was activated by fusion radiation, and was demonstrated to be important in the enhanced expression of HO-1 and COX-2 through stimulating the activation of $\mathrm{p} 38 \mathrm{MAPK}$. The results of the present 
study provide novel information on fusion radiation-induced biological effects and potential targets for decreasing the health risks.

\section{Acknowledgements}

The authors would like to thank the other members of the Fusion Design and Study team for their assistance in the present study.

\section{Funding}

The present study was supported by the National Magnetic Confinement Fusion Science Program of China (grant no. 2014GB112006), the Natural Science Foundation of Anhui Province of China (grant no. 1508085SME220), the National Natural Science Foundation of China (grant nos. 11575232 and 31370842), the International Partnership Program of Chinese Academy of Sciences (grant no. 116134KYSB20160084) and the Innovative Program of Development Foundation of Hefei Center for Physical Science and Technology (grant no. 2016FXCX005).

\section{Availability of data and materials}

The analyzed data sets during the study are available from the corresponding author upon reasonable request.

\section{Authors' contributions}

JW, XY and HL designed the experiments. XY, HL and XJ performed the biological experiments. CJ, ZX, TL and $\mathrm{ZW}$ performed the deuterium-tritium fusion radiation of biological samples. XY, HL and JW analyzed the results and wrote the manuscript. All authors read and approved the final manuscript.

\section{Ethics approval and consent to participate}

Not applicable.

\section{Patient consent for publication}

Not applicable.

\section{Competing interests}

The authors declare that they have no competing interests.

\section{References}

1. Lomax ME, Folkes LK and O'Neill P: Biological consequences of radiation-induced DNA damage: Relevance to radiotherapy. Clin Oncol (R Coll Radiol) 25: 578-585, 2013.

2. Najafi M, Motevaseli E, Shirazi A, Geraily G, Rezaeyan A, Norouzi F, Rezapoor S and Abdollahi H: Mechanisms of inflammatory responses to radiation and normal tissues toxicity: Clinical implications. Int J Radiat Biol 94: 335-356, 2018.

3. Gozzelino R, Jeney V and Soares MP: Mechanisms of cell protection by heme oxygenase-1. Annu Rev Pharmacol Toxicol 50: 323-354, 2010.
4. Vile GF, Basu-Modak S, Waltner C and Tyrrell RM: Heme oxygenase 1mediates an adaptive response to oxidative stress in human skin fibroblasts. Proc Natl Acad Sci USA 91: 2607-2610, 1994.

5. Otterbein LE and Choi AM: Heme oxygenase: Colors of defense against cellular stress. Am J Physiol Lung Cell Mol Physiol 279: L1029-L1037, 2000.

6. Ryter SW, Alam J and Choi AM: Heme oxygenase-1/carbon monoxide: From basic science to therapeutic applications. Physiol Rev 86: 583-650, 2006.

7. Soares MP, Marguti I, Cunha A and Larsen R: Immunoregulatory effects of HO-1: How does it work? Curr Opin Pharmacol 9: 482-489, 2009.

8. Paine A, Eiz-Vesper B, Blasczyk R and Immenschuh S: Signaling to heme oxygenase-1 and its anti-inflammatory therapeutic potential. Biochem Pharmacol 80: 1895-1903, 2010.

9. Pang LY, Hurst EA and Argyle DJ: Cyclooxygenase-2: A role in cancer stem cell survival and repopulation of cancer cells during therapy. Stem Cells Int 2016: 2048731, 2016.

10. Tang FR and Loke WK: Molecular mechanisms of low dose ionizing radiation-induced hormesis, adaptive responses, radioresistance, bystander effects, and genomic instability. Int J Radiat Biol 91: 13-27, 2015.

11. Salehifar E and Hosseinimehr SJ: The use of cyclooxygenase-2 inhibitors for improvement of efficacy of radiotherapy in cancers. Drug Discov Today 21: 654-662, 2016.

12. Rosser CJ, Gaar M and Porvasnik S: Molecular fingerprinting of radiation resistant tumors: Can we apprehend and rehabilitate the suspects? BMC Cancer 9: 225, 2009.

13. Komaki R, Liao Z and Milas L: Improvement strategies for molecular targeting: Cyclooxygenase-2 inhibitors as radiosensitizers for non-small cell lung cancer. Semin Oncol 31 (Suppl 1): S47-S53, 2004.

14. Nie B, Ni M and Wei S: Individual dose due to radioactivity accidental release from fusion reactor. J Hazard Mater 327: 135-143, 2017.

15. Wu Y: Development of high intensity D-T fusion neutrongenerator HINEG. Int J Energy Res 42: 68-72, 2016.

16. Bustin SA, Benes V, Garson JA, Hellemans J, Huggett J, Kubista M, Mueller R, Nolan T, Pfaffl MW, Shipley GL, et al: The MIQE guidelines: Minimum information for publication of quantitative real-time PCR experiments. Clin Chem 54: 611-622, 2009.

17. Tran V and Little MP: Dose and dose rate extrapolation factors for malignant and non-malignant health endpoints after exposure to gamma and neutron radiation. Radiat Environ Biophys 56: 299-328, 2017.

18. Broustas CG, Xu Y, Harken AD, Chowdhury M, Garty G and Amundson SA: Impact of neutron exposure on global gene expression in a human peripheral blood model. Radiat Res 187: 433-440, 2017.

19. Tanaka K, Gajendiran N, Endo S, Komatsu K, Hoshi M and Kamada N: Neutron energy-dependent initial DNA damage and chromosomal exchange. J Radiat Res 40 (Suppl): S36-S44, 1999.

20. Otterbein LE, Hedblom A, Harris C, Csizmadia E, Gallo D and Wegiel B: Heme oxygenase-1 and carbon monoxide modulate DNA repair through ataxia-telangiectasia mutated (ATM) protein. Proc Natl Acad Sci USA 108: 14491-14496, 2011.

21. Chen N, Wu L, Yuan H and Wang J: ROS/Autophagy/Nrf2 pathway mediated low-dose radiation induced radio-resistance in human lung adenocarcinoma A549 cell. Int J Biol Sci 11: 833-844, 2015.

22. Han W, Yu KN, Wu LJ, Wu YC and Wang HZ: Mechanism of protection of bystander cells by exogenous carbon monoxide: Impaired response to damage signal of radiation-induced bystander effect. Mutat Res 709-710: 1-6, 2011.

23. Singh A, Yashavarddhan MH, Kalita B, Ranjan R, Bajaj S, Prakash H and Gupta ML: Podophyllotoxin and Rutin modulates ionizing radiation-induced oxidative stress and apoptotic cell death in mice bone marrow and spleen. Front Immunol 8: 183, 2017.

24. Hofer M, Hoferová Z, Dušek L, Souček K and Gruzdev A: Hematological profile of untreated or ionizing radiation-exposed cyclooxygenase-2-deficient mice. Physiol Res 66: 673-676, 2017.

25. Ozbilgin MK, Onal T, Ozcan C, Temel M, Aktas C, Gareveran MS, Uluer ET, Inan S and Kurtman C: Effects of cyclooxygenase on the urothelium of the urinary bladder of mice exposed to pelvic radiation. Anal Quant Cytopathol Histpathol 38: 103-110, 2016. 
26. Zuo Z, Ouyang W, Li J, Costa M and Huang C: Cyclooxygenase-2 (COX-2) mediates arsenite inhibition of UVB-induced cellular apoptosis in mouse epidermal Cl41 cells. Curr Cancer Drug Targets 12: 607-616, 2012.

27. Clouaire T, Marnef A and Legube G: Taming tricky DSBs: ATM on duty. DNA Repair (Amst) 56: 84-91, 2017.

28. Thompson LH: Recognition, signaling, and repair of DNA double-strand breaks produced by ionizing radiation in mammalian cells: The molecular choreography. Mutat Res 751: 158-246, 2012.

29. Park SY, Kim YM and Pyo H: Gefitinib radiosensitizes non-small cell lung cancer cells through inhibition of ataxia telangiectasia mutated. Mol Cancer 9: 222, 2010.

30. Chacko T, Menon A, Majeed T, Nair SV, John NS and Nair CKK Mitigation of whole-body gamma radiation-induced damages by Clerodendron infortunatum in mammalian organisms. J Radiat Res 58: 281-291, 2017.

31. Kim YM and Pyo H: Cooperative enhancement of radiosensitivity after combined treatment of 17-(allylamino)-17-demethoxygeldanamycin and celecoxib in human lung and colon cancer cell lines. DNA Cell Biol 31: 15-29, 2012.

32. Dent P, Yacoub A, Fisher PB, Hagan MP and Grant S: MAPK pathways in radiation responses. Oncogene 22: 5885-5896, 2003.

33. Munshi A and Ramesh R: Mitogen-activated protein kinases and their role in radiation response. Genes Cancer 4: 401-408, 2013.
34. Acheva A, Schettino G and Prise KM: Pro-inflammatory signaling in a 3D organotypic skin model after low LET irradiation-NF- $\mathrm{kB}, \mathrm{COX}-2$ activation, and impact on cell differentiation. Front Immunol 8: 82, 2017.

35. Hung JH, Su IJ, Lei HY, Wang HC, Lin WC, Chang WT, Huang W, Chang WC, Chang YS, Chen CC and Lai MD: Endoplasmic reticulum stress stimulates the expression of cyclooxygenase-2 through activation of NF-kappaB and pp38 mitogen-activated protein kinase. J Biol Chem 279: 46384-46392, 2004.

36. Tessner TG, Muhale F, Schloemann S, Cohn SM, Morrison AR and Stenson WF: Ionizing radiation up-regulates cyclooxygenase-2 in 1407 cells through p38 mitogen-activated protein kinase. Carcinogenesis 25: 37-45, 2004

37. Hu YP, Peng YB, Zhang YF, Wang Y, Yu WR, Yao M and $\mathrm{Fu}$ XJ: Reactive oxygen species mediated prostaglandin E2 contributes to acute response of epithelial injury. Oxid Med Cell Longev 2017: 4123854, 2017.

(i)(3) This work is licensed under a Creative Commons Attribution-NonCommercial-NoDerivatives 4.0 International (CC BY-NC-ND 4.0) License. 